\title{
Connecting QCD to neutrino-nucleus scattering
}

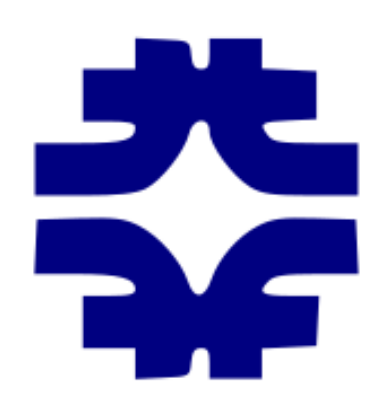

Fermilab

\author{
Michael Wagman
}

Snowmass Mini-Workshop on

Neutrino Theory

Sep 23, 2020

Connecting QCD to neutrino-nucleus scattering Lol link

Joseph Carlson ${ }^{1}$, Chia Cheng Chang (張家丞) ${ }^{2,3,4}$, William Detmold ${ }^{5}$, Joshua Isaacson $^{6}$, William Jay ${ }^{6}$, Gurtej Kanwar ${ }^{5}$, Andreas Kronfeld ${ }^{6}$, Huey-Wen Lin ${ }^{7}$, Yin Lin (林胤 $)^{6,8}$, Keh-Fei Liu ${ }^{9}$, Alessandro Lovato ${ }^{10,11}$, Pedro Machado ${ }^{6}$, Aaron S. Meyer ${ }^{12}$, Saori Pastore $^{13}$, Noemi Rocco ${ }^{6,10}$, Phiala Shanahan ${ }^{5}$, and Michael Wagman 6 


\section{The precision era of $\nu$ physics}

Next-generation oscillation experiments can answer fundamental questions about the neutrino mass ordering, size of leptonic $C P$ violation, and more

Discovering new physics with $\nu$ experiments requires control of all the Standard Model physics in the detector

Acciarri et al (DUNE) arXiv 1512.06148

$$
\underbrace{N_{\mathrm{FD}}^{\text {expected }}\left(\nu_{e}\right)}_{\text {Measured }}=\underbrace{N_{\mathrm{ND}}^{\text {data }}\left(\nu_{\mu}\right)}_{\nu A \text { cross-section }} \otimes \frac{\Phi_{\mathrm{FD}}\left(\nu_{\mu}\right)}{\Phi_{\mathrm{ND}}\left(\nu_{\mu}\right)} \otimes \underbrace{P\left(\nu_{\mu} \rightarrow \nu_{e}\right)}_{\text {Goal }} \otimes \underbrace{\varepsilon_{\mathrm{FD}}\left(\nu_{\nu}\right)}_{\varepsilon_{\mathrm{ND}}\left(\nu_{\mu}\right)} \otimes \underbrace{}_{\frac{\sigma_{\mathrm{FD}}\left(\nu_{e}\right)}{\sigma_{\mathrm{ND}}\left(\nu_{\mu}\right)}}
$$

Exclusive $\nu A$ cross-sections required for event-by-event reconstruction of incident neutrino energy

See talks by Shirley Li, Will Jay, Josh Barrow, Kajetan Niewczas

Sanford

Underground

Research

Facility

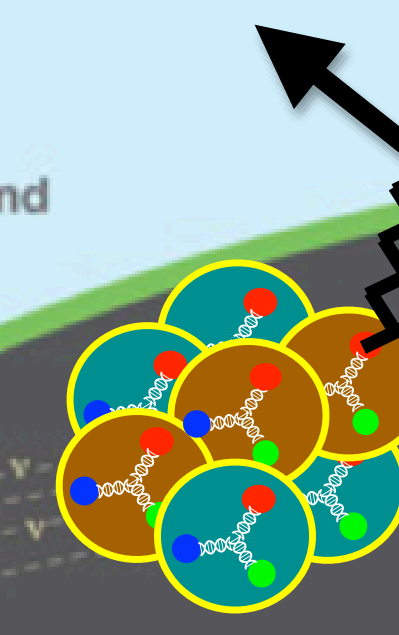

Fermilab

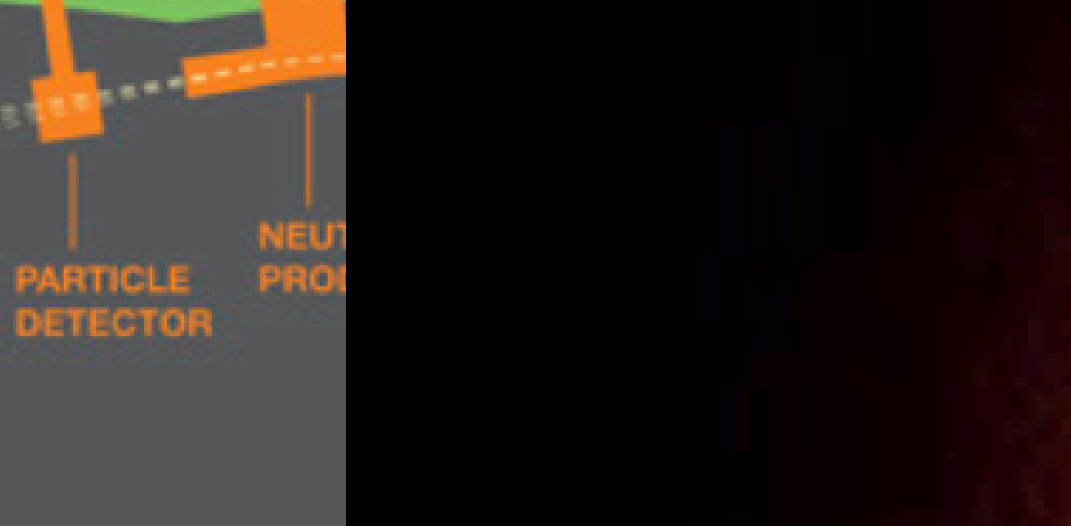




\section{Challenges of $\nu A$}

Neutrino-argon cross-sections with few percent-level accuracy required to achieve design sensitivity to $C P$ violation at DUNE

Acciarri et al (DUNE) arXiv 1512.06148
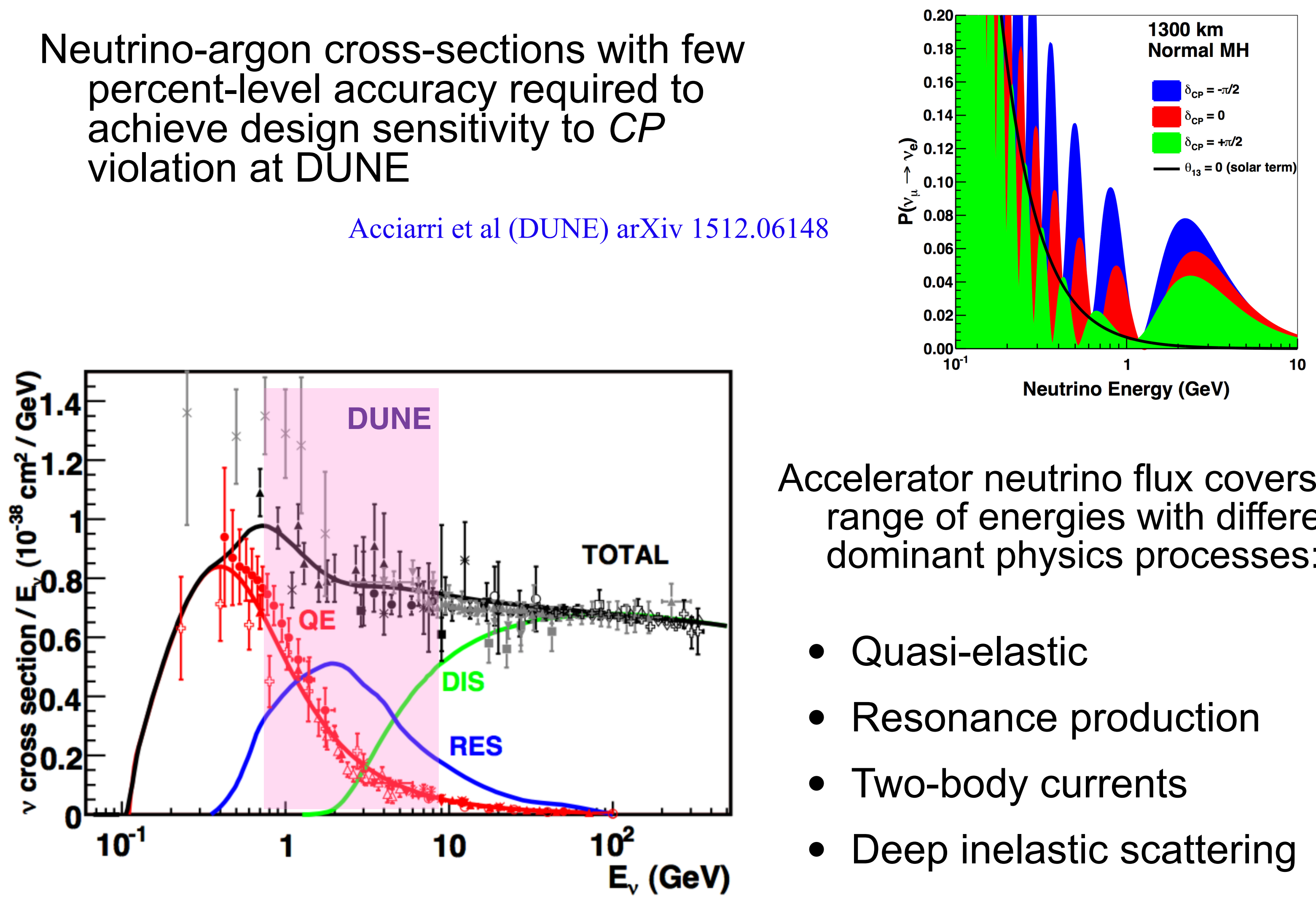

Accelerator neutrino flux covers a wide range of energies with different dominant physics processes:

- Quasi-elastic

- Resonance production

- Two-body currents

- Deep inelastic scattering 


\section{Lattice QCD and $\nu A$}

$\nu A$ scattering amplitudes factorize into leptonic and hadronic parts

$$
\mathcal{M}_{\nu A \rightarrow \ell f} \propto\left(\bar{u}_{\ell} \gamma_{\mu} \gamma_{5} P_{L} u_{\nu}\right)\left\langle f\left|\bar{q} \gamma_{\mu} \gamma_{5} P_{L} q\right| A\right\rangle
$$

Euclidean hadronic matrix elements calculable (in principle) using lattice QCD

$\langle\mathcal{O}\rangle=\int \mathcal{D} U \mathcal{D} \bar{q} \mathcal{D} q e^{-S_{Q C D}(U, q, \bar{q})} \mathcal{O}(U, q, \bar{q}) \approx \frac{1}{N_{\text {cfg }}} \sum_{i=1}^{N_{\text {cfg }}} \mathcal{O}\left(U_{i}\right)$

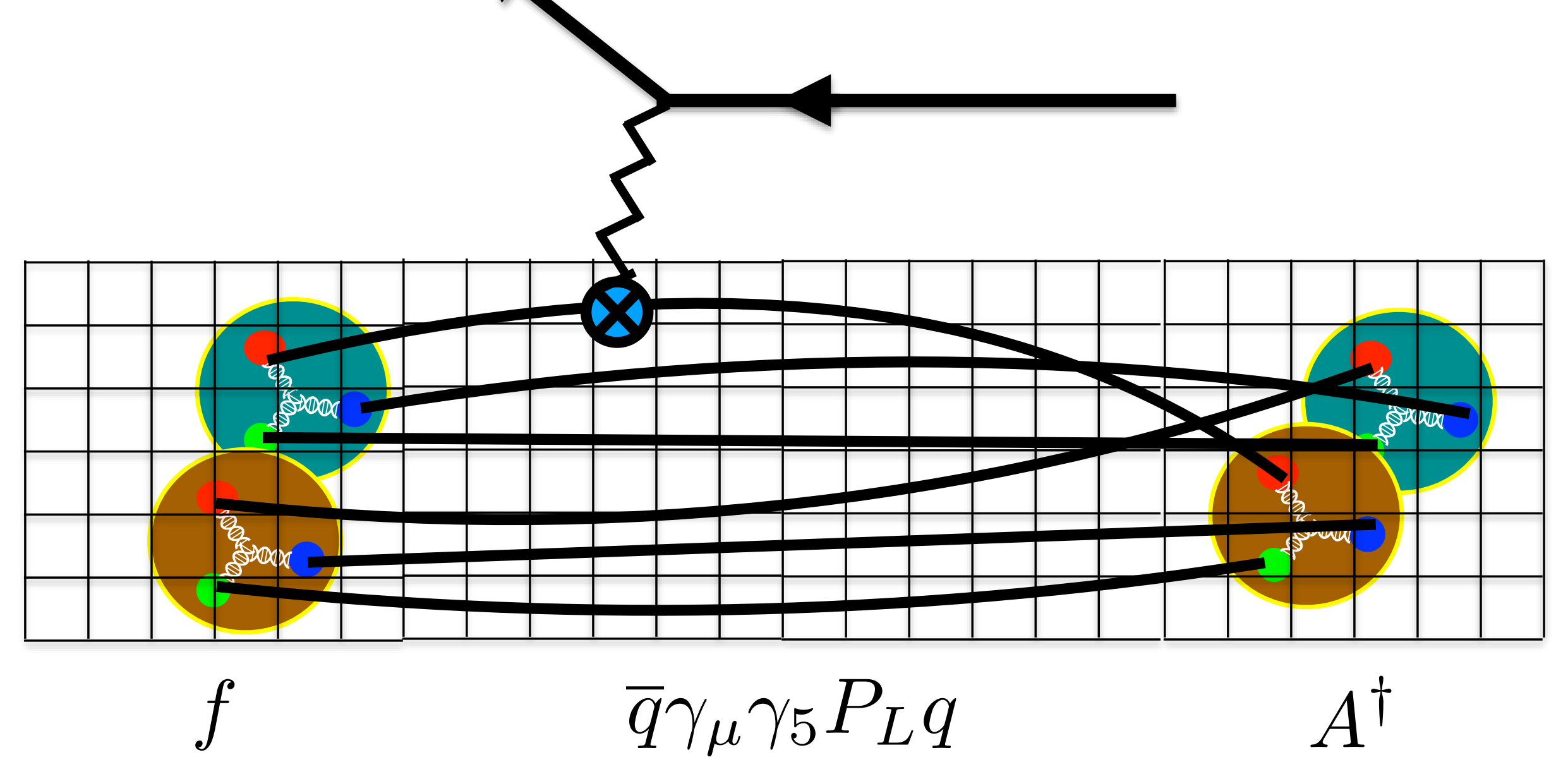




\section{Lattice QCD, EFT, and $\nu A$}

Lattice QCD simulations of nuclei face several practice challenges:

- Exponential signal-to-noise degradation

- Small gaps to finite-volume excited states

- Rapid growth in number of Wick contractions

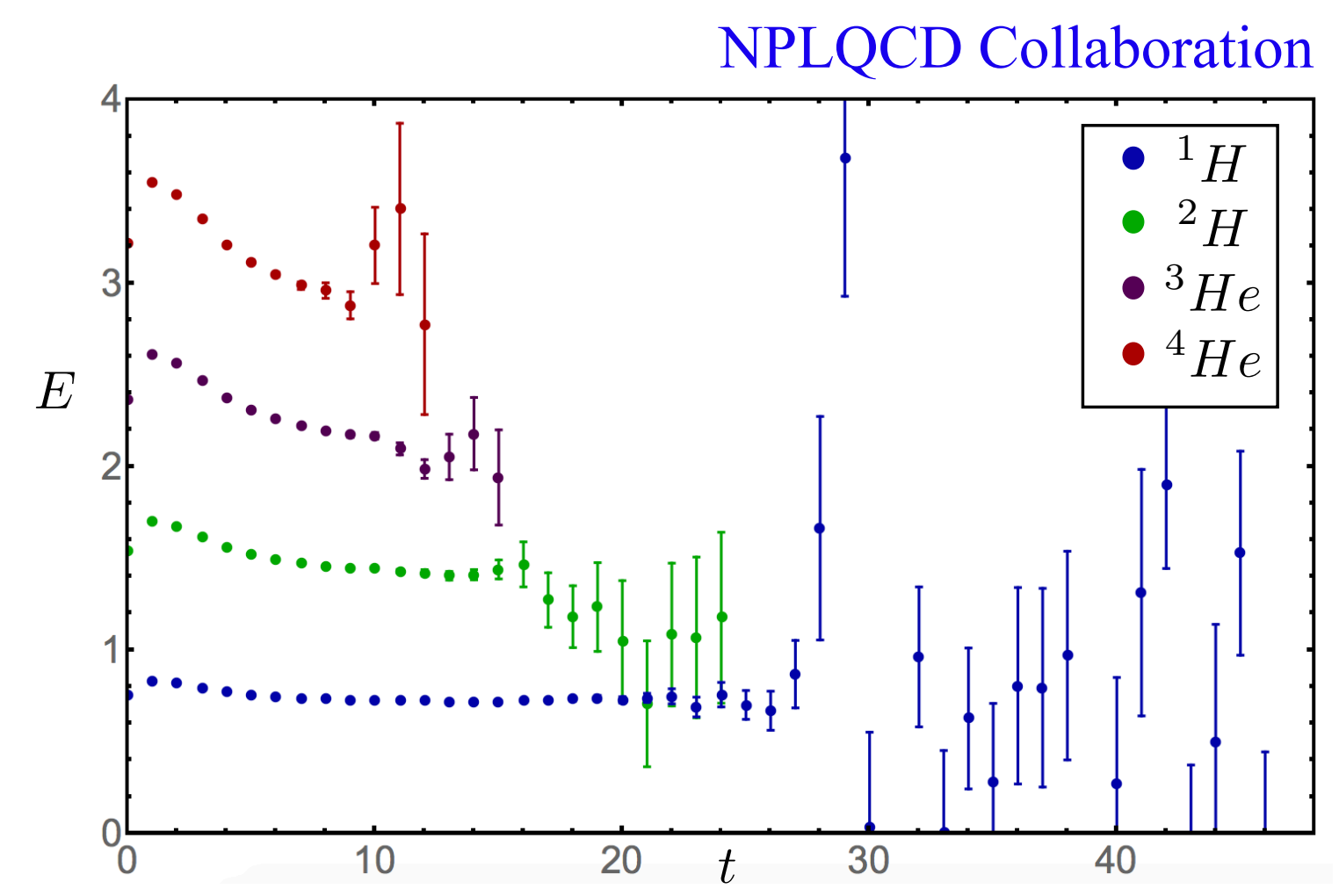

Matrix element studies so far limited to $A \leq 3$ with unphysical quark masses

Review: Davoudi, Detmold, Orginos, Parreño, Savage, Shanahan, MW arXiv:2008.11160

Nuclear many-body methods are capable of efficiently describing larger nuclei, but require accurate inputs that are not all known from experiment

See talk by Noemi Rocco

LQCD

Nuclear EFT and many-body

\section{Event generators}




\section{Quasi-elastic region}

Nucleons are effective degrees of freedom for nuclei at low energies

Currents dominantly couple to single-nucleon operators

Impulse approximation:

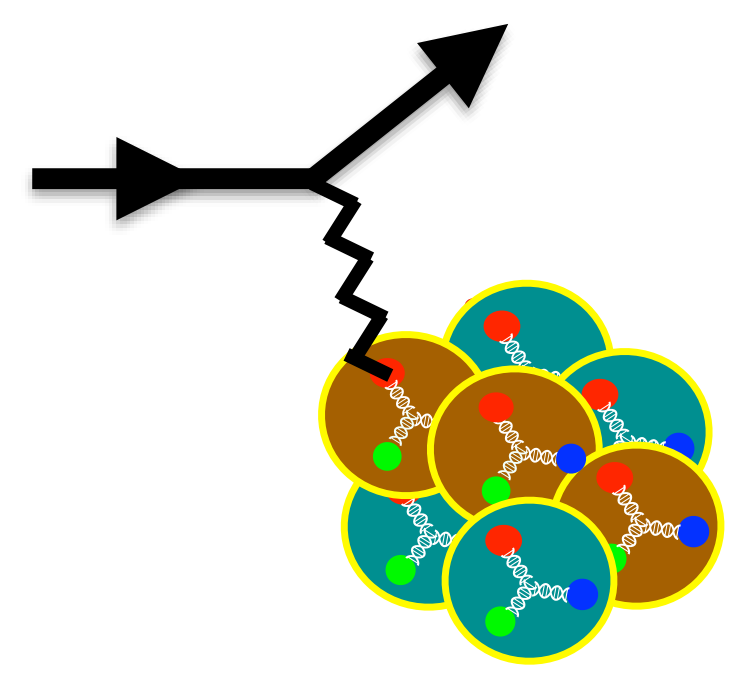

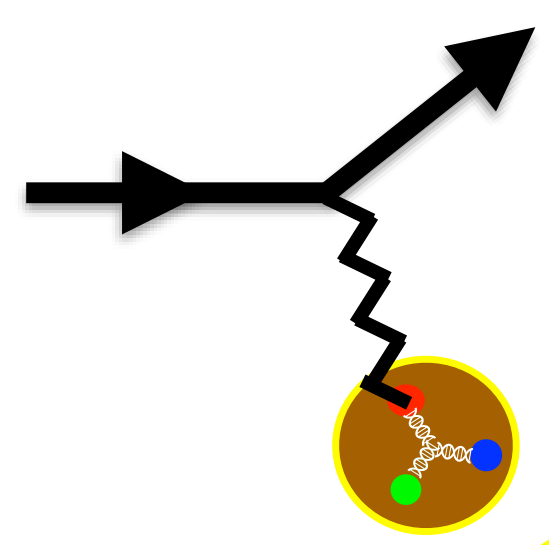

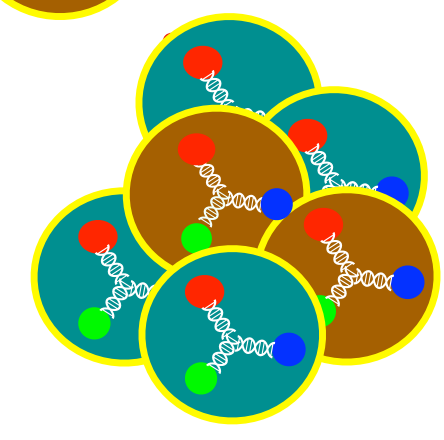

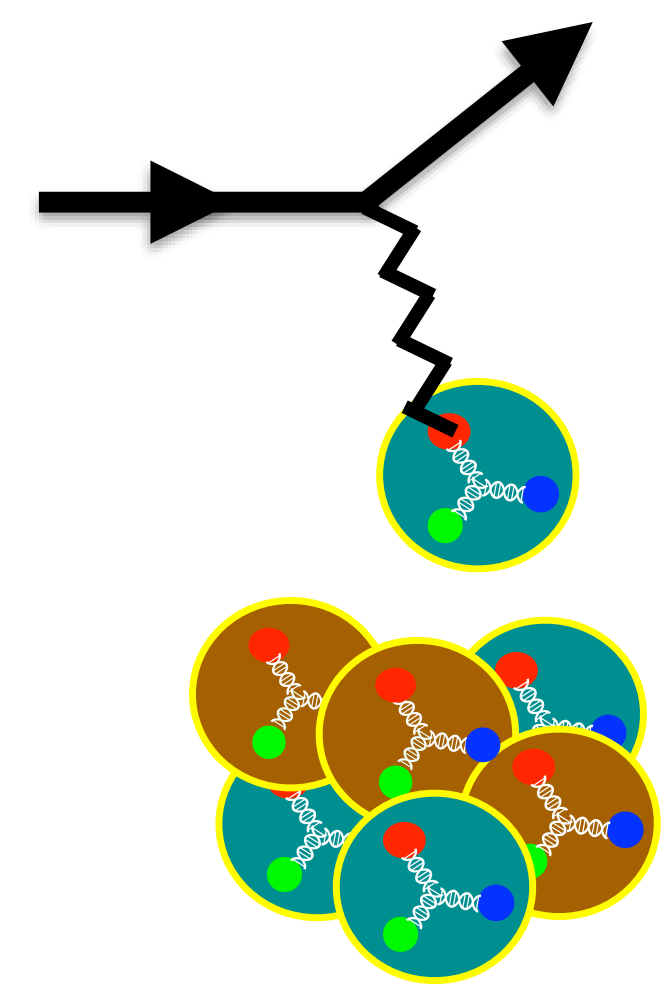

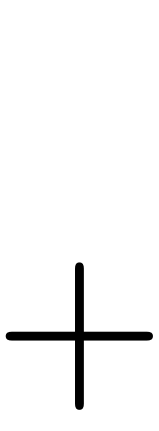

Cross-section in this region controlled by single-nucleon form factors

Vector current form factors are constrained by electron scattering, axial current form factors are less well-known 


\section{Single-nucleon QCD input}

Axial charge precisely determined from LQCD with controlled uncertainties by multiple groups

$$
\begin{aligned}
& J_{A}^{3}(\mathbf{q})=\sum_{\mathbf{x}} e^{i \mathbf{q} \cdot \mathbf{x}} \bar{q}(\mathbf{x}) \gamma_{z} \gamma_{5} \tau^{3} q(\mathbf{x}) \\
& g_{A}=\left\langle p\left|J_{A}^{3}(0)\right| p\right\rangle
\end{aligned}
$$
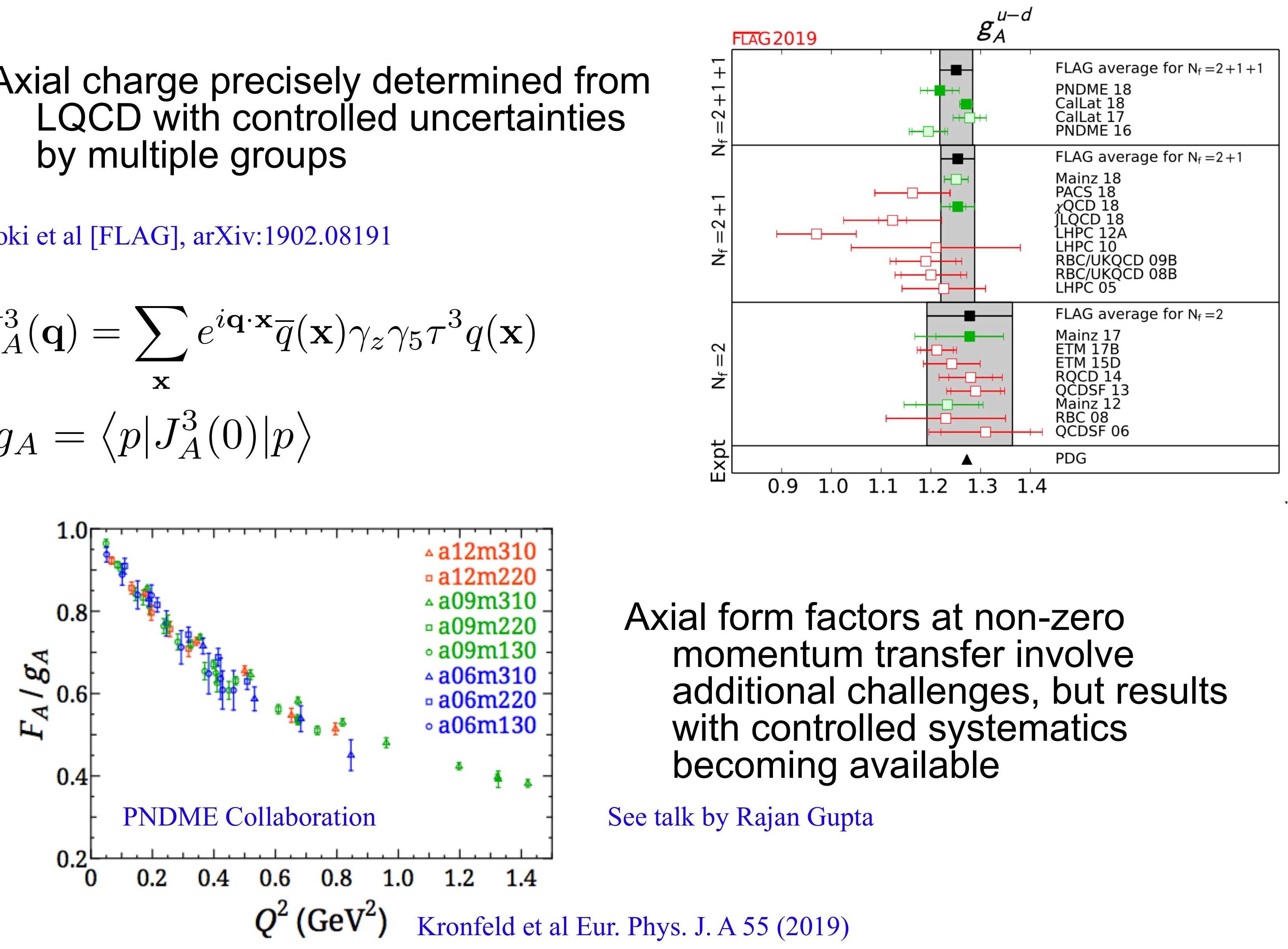


\section{Resonance region}

At higher energies, pions and other resonances can be produced

Exclusive pion production and scattering processes must be modeled in event generators

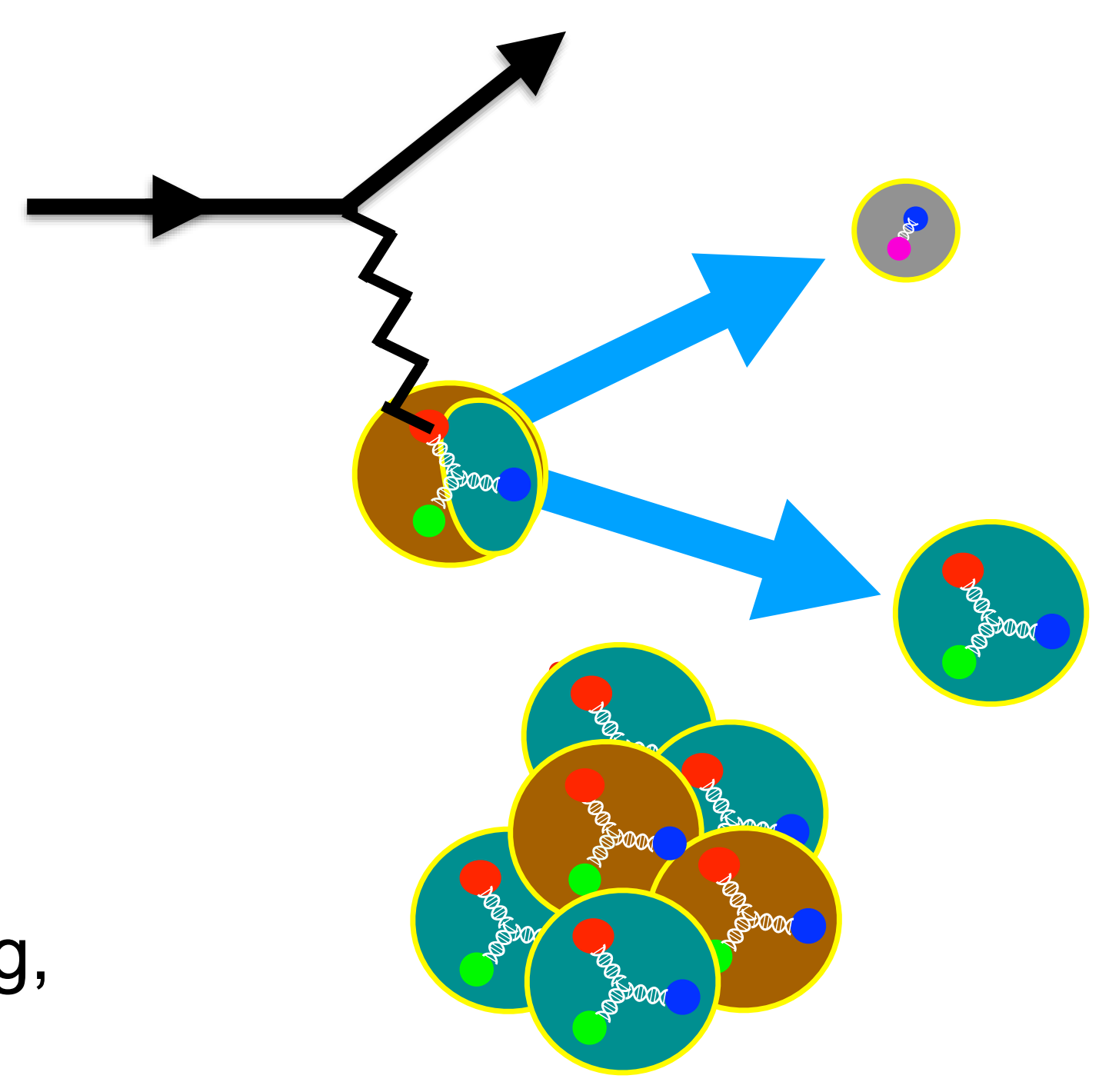

Including explicit pions in EFT in a consistent power counting is challenging, still unresolved for $A \gtrsim 3$

Review: van Kolck, Front. In Phys. 8 (2020)

See talks by A. Nicholson and E. Mereghetti

Accurate benchmarks from experiment + LQC D are essential for validating nuclear models without controlled power counting 


\section{Finite-volume physics}

Pion production and scattering are dynamical processes that can not be determined from infinite-volume Euclidean observables

Solution - exploit the finite volume used in simulations

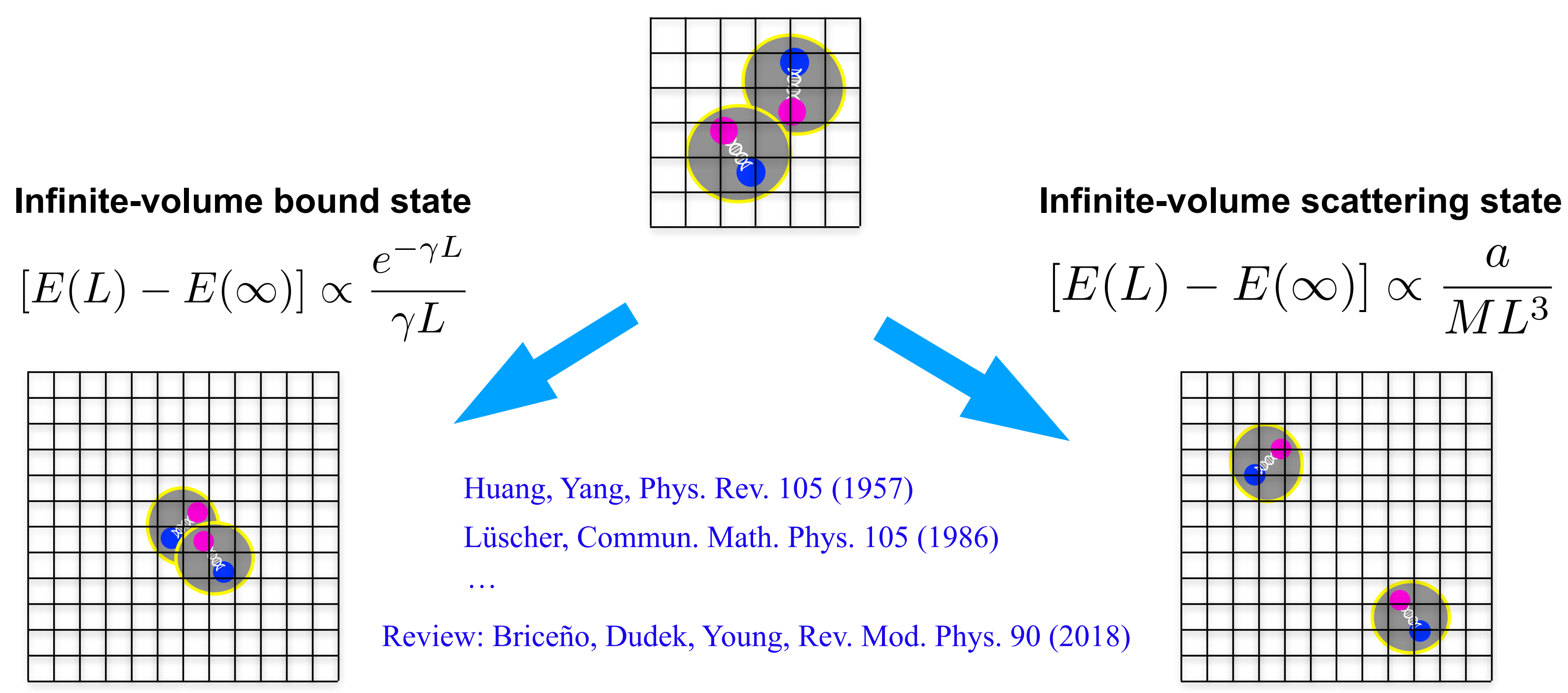

Extensions to finite-volume resonant form factors: Baroni, Briceño, Hansen, Ortega-Gama, 


\section{Shallow inelastic region}

Correlated interactions of nucleon pairs are important at higher energies

Pionless EFT:
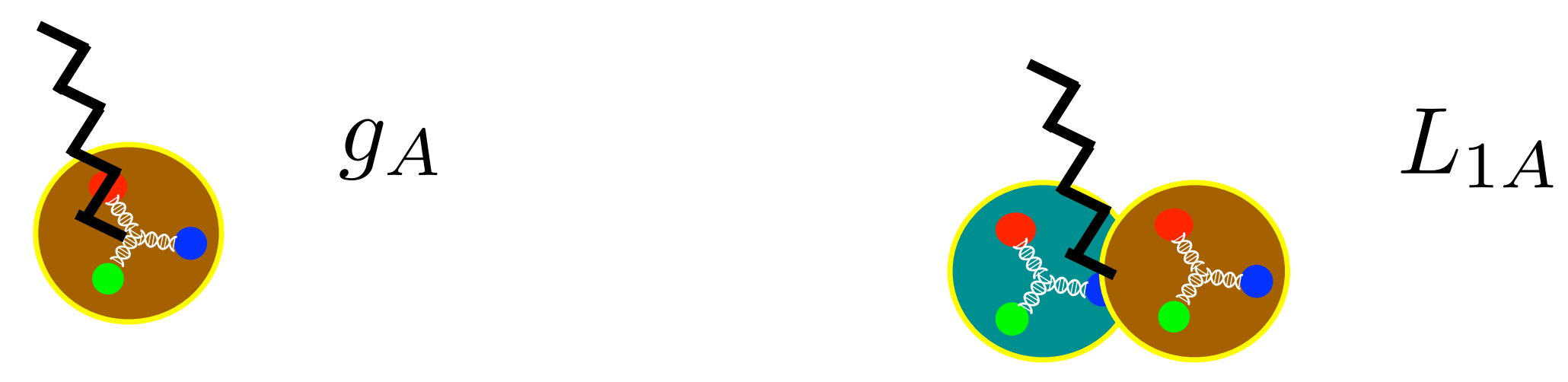

Similar two-body current operators arise in chiral EFT and other nuclear many-body formalisms

Benchmarks of two-body current effects will be essential for constraining and validating phenomenological nuclear models using spectral functions etc.

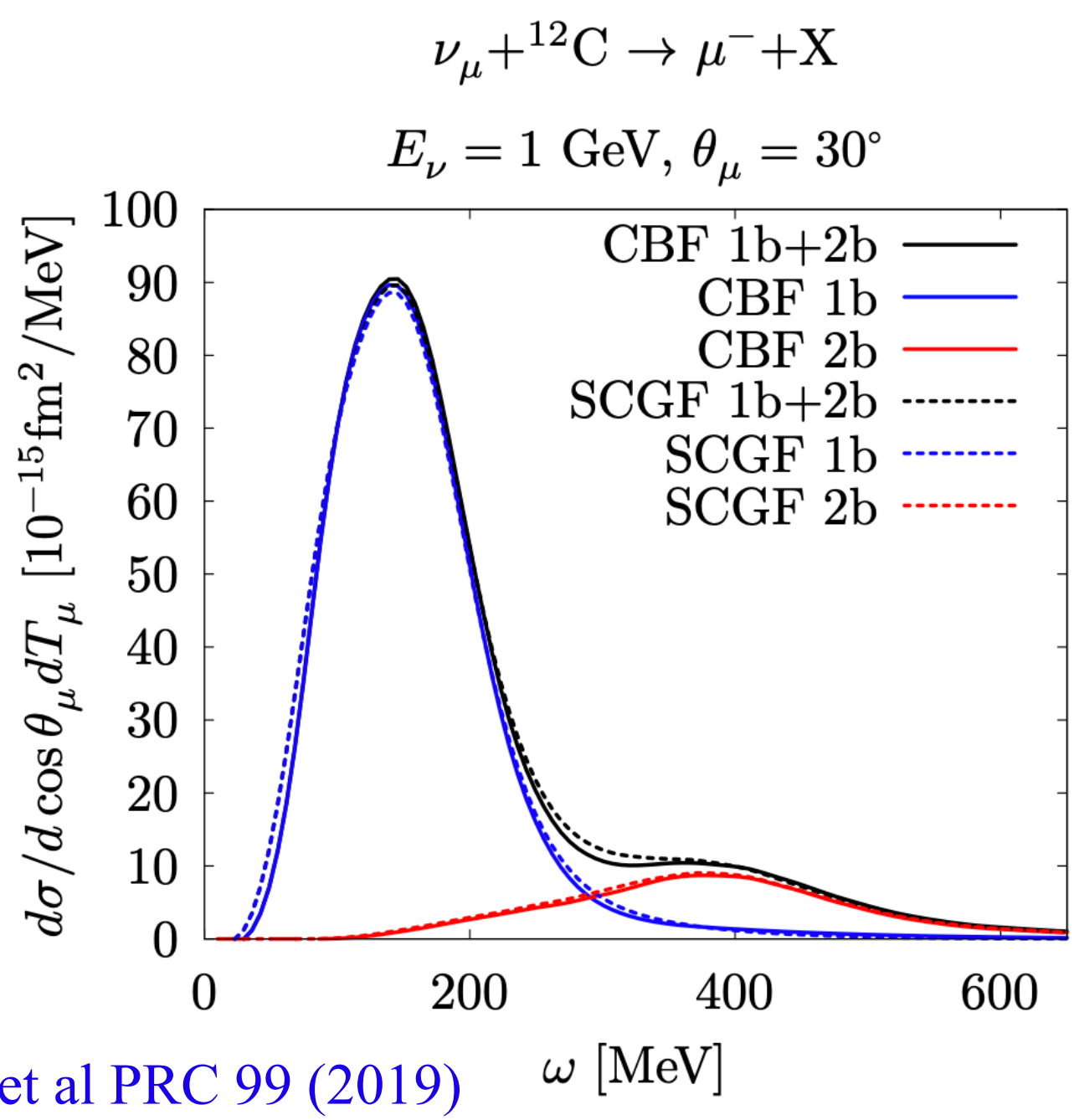




\section{Constraining two-body currents}

First constraints on two-body axial currents obtained by matching LQCD and EFT calculations in a box with a background axial field
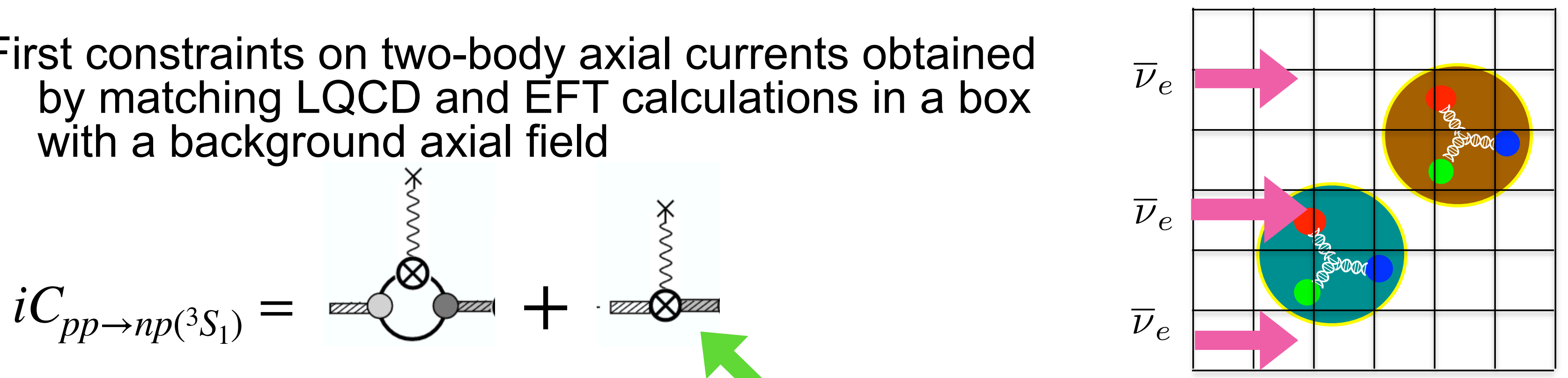

$$
\mathscr{M}_{p p \rightarrow n p\left({ }^{3} S_{1}\right)}=g A(1+S)-L_{1 A}
$$

Short-distance QCD

physics

Used for LQCD determination of $L_{1 A}$ and constraints on proton-proton fusion

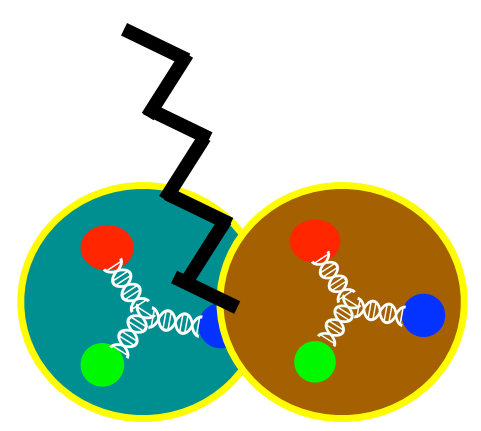

$L_{1 A}=3.9(0.2)(1.0)(0.4)(0.9) \mathrm{fm}^{3}$

Savage et al [NPLQCD], PRL 119 (2017)

\begin{tabular}{lcc}
\hline & method & $L_{1 A}\left(\mathrm{fm}^{3}\right)$ \\
\hline two-body & & \\
& reactor $\bar{\nu}+d$ & $3.6 \pm 5.5[11]$ \\
& ES, CC, NC in SNO & $4.0 \pm 6.3[41]$ \\
MuSun proposal & $\pm \mathbf{1 . 2 5}$ \\
\hline \hline three-body & tritium beta decay & $4.2 \pm 3.7[11], 4.2 \pm 0.1[41]$ \\
\hline other & helioseismology & $4.8 \pm 6.7[42]$ \\
&
\end{tabular}

Andreev et al (MuSun) arXiv 1004.1754 


\section{Two-body current effects}

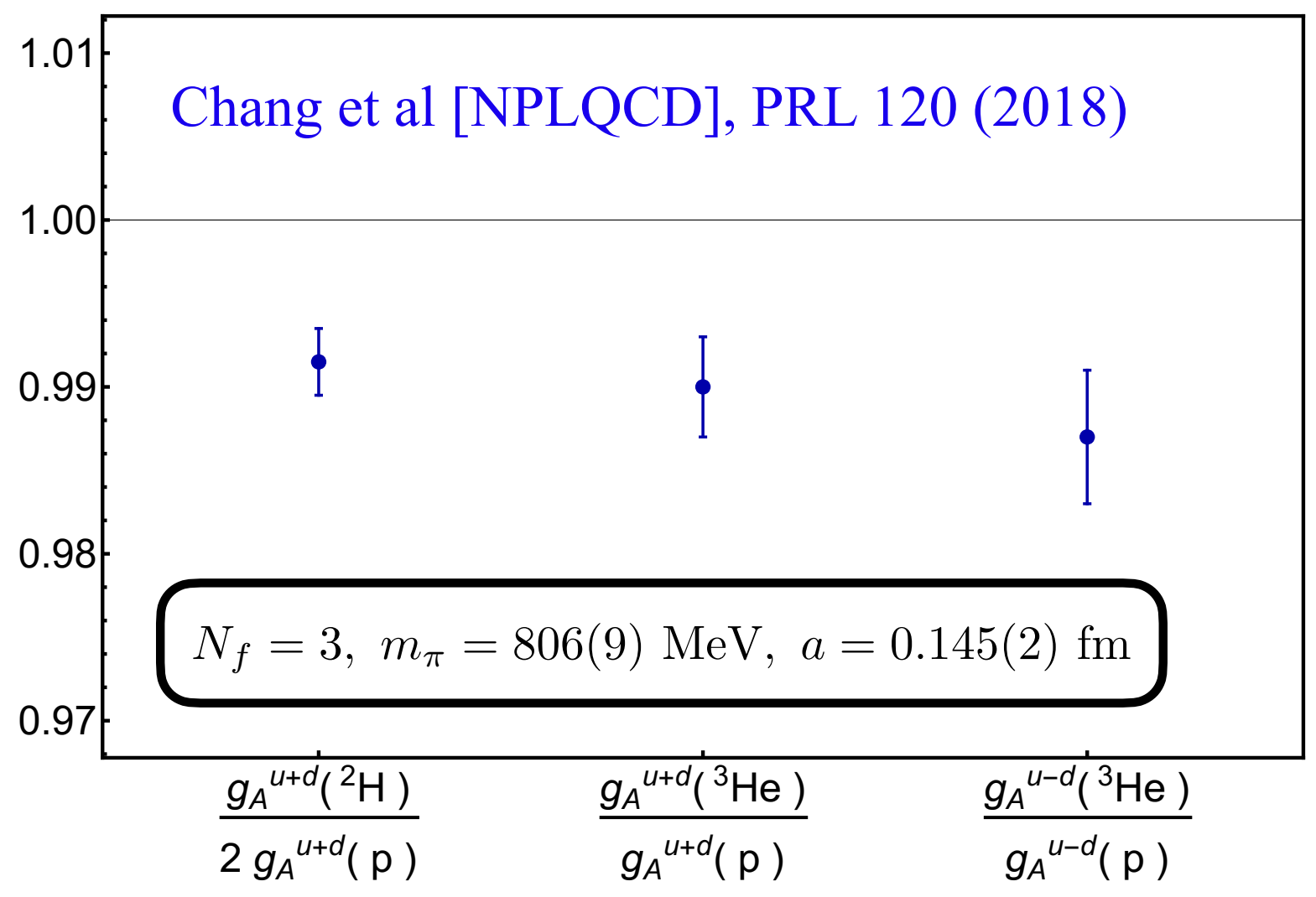

Nuclear modifications of $u$ and $d$ quark axial couplings $\mathrm{O}(1 \%)$ for $\mathrm{A}=2-3$ nuclei, $m_{\pi} \sim 806 \mathrm{MeV}$

QCD effects reduce axial charge of ${ }^{3} \mathrm{H}$ by $4.89(13) \%$ in nature

$A=30-50$ nuclei have $O(30 \%)$ smaller axial couplings than predicted by shell model with single-particle couplings

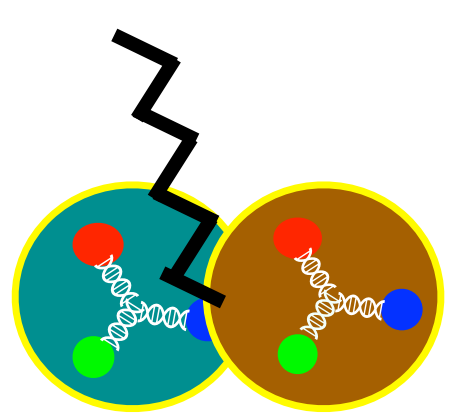

Multi-nucleon correlations and currents needed to reproduce $\beta$-decay results from experiment

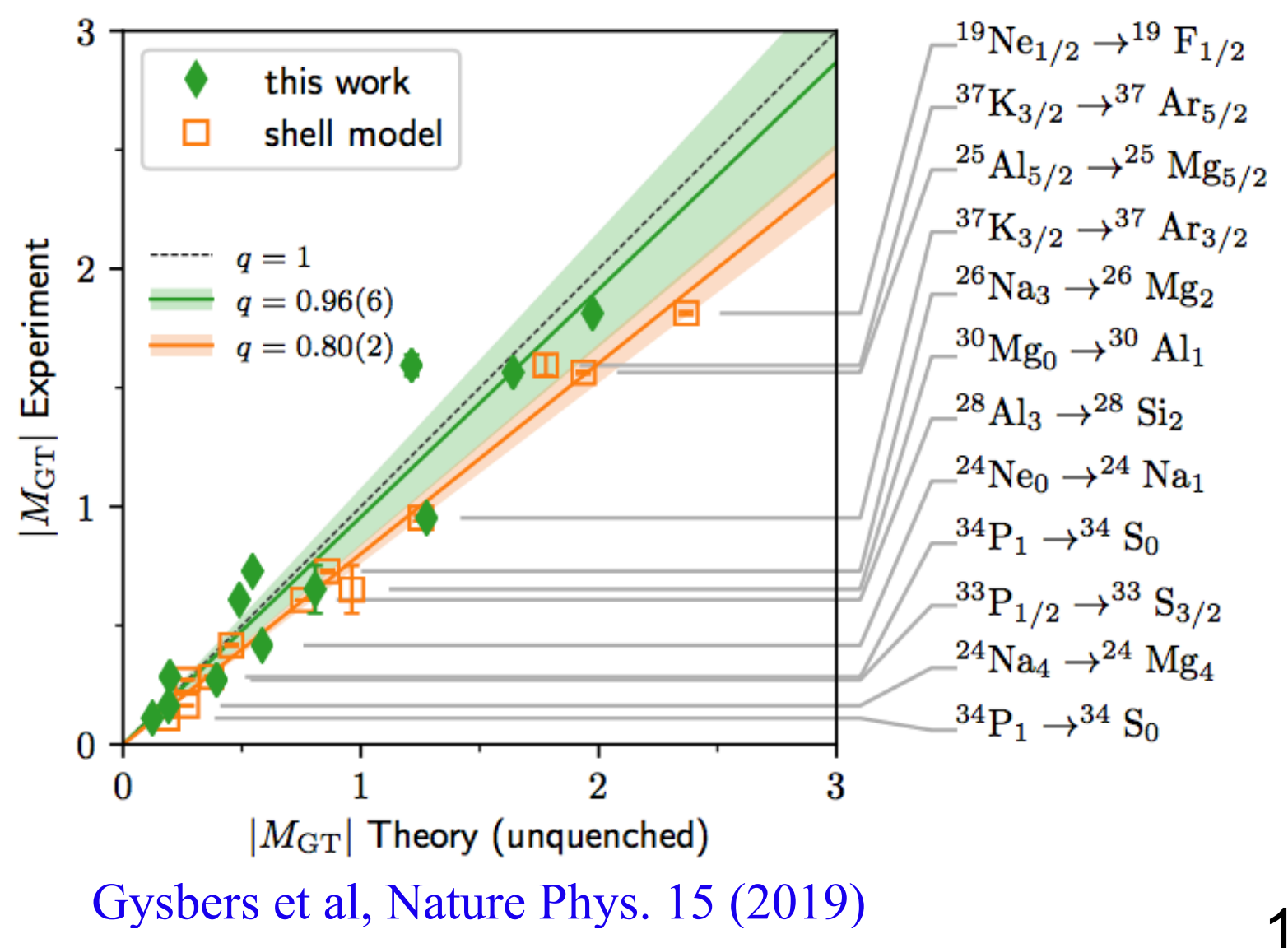




\section{Deep inelastic regime}

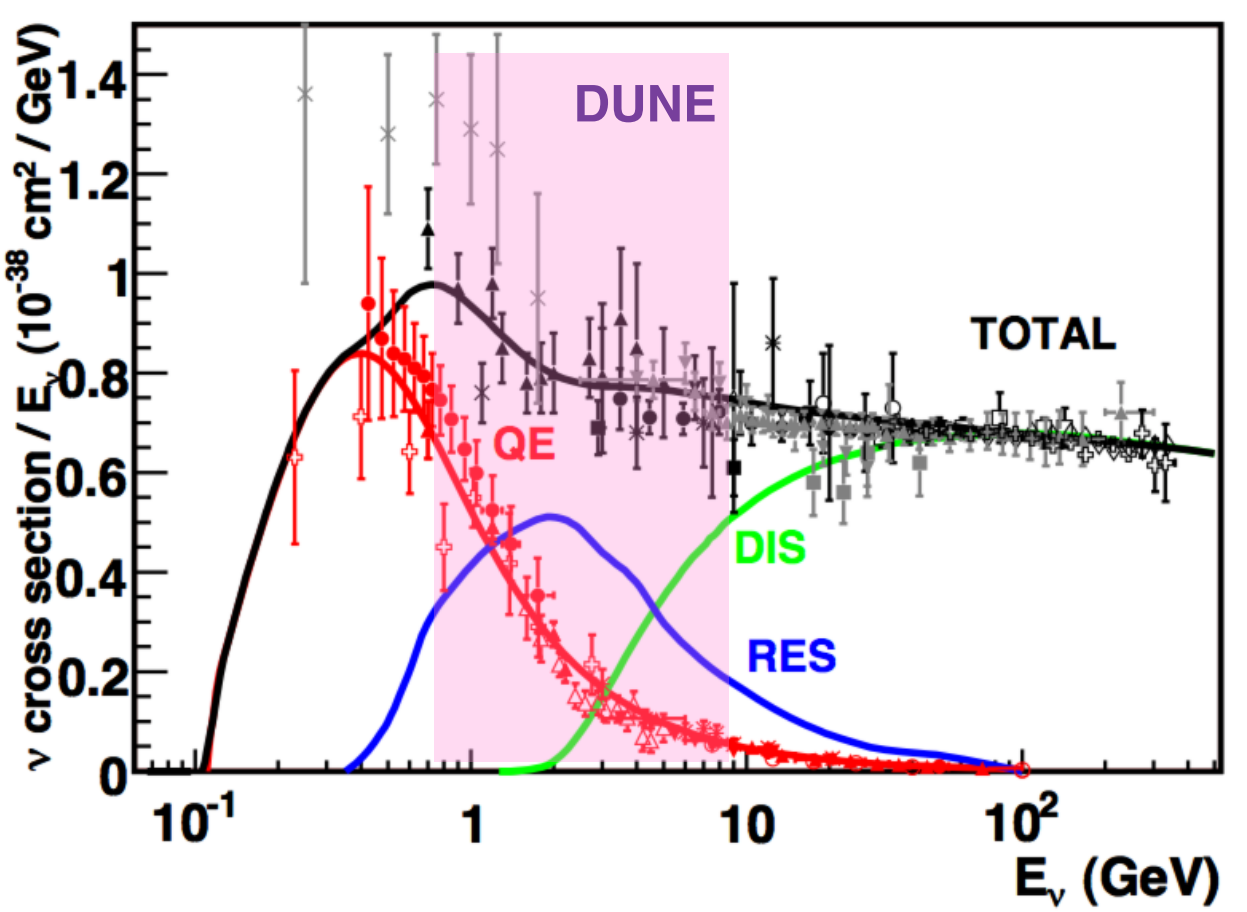

Adapted from Formaggio, Zeller, Rev. Mod. Phys. 84 (2012)

Nonperturbative QCD effects determine the PDFs themselves

Some aspects of PDFs are experimentally well-known, but others (e.g. flavor dependence, nuclear effects) are not
At very high energies, cross-sections factorize into convolutions of perturbative quark-and-gluon-level amplitudes and nonperturbative PDFs

Including perturbative QCD effects in event generators essential

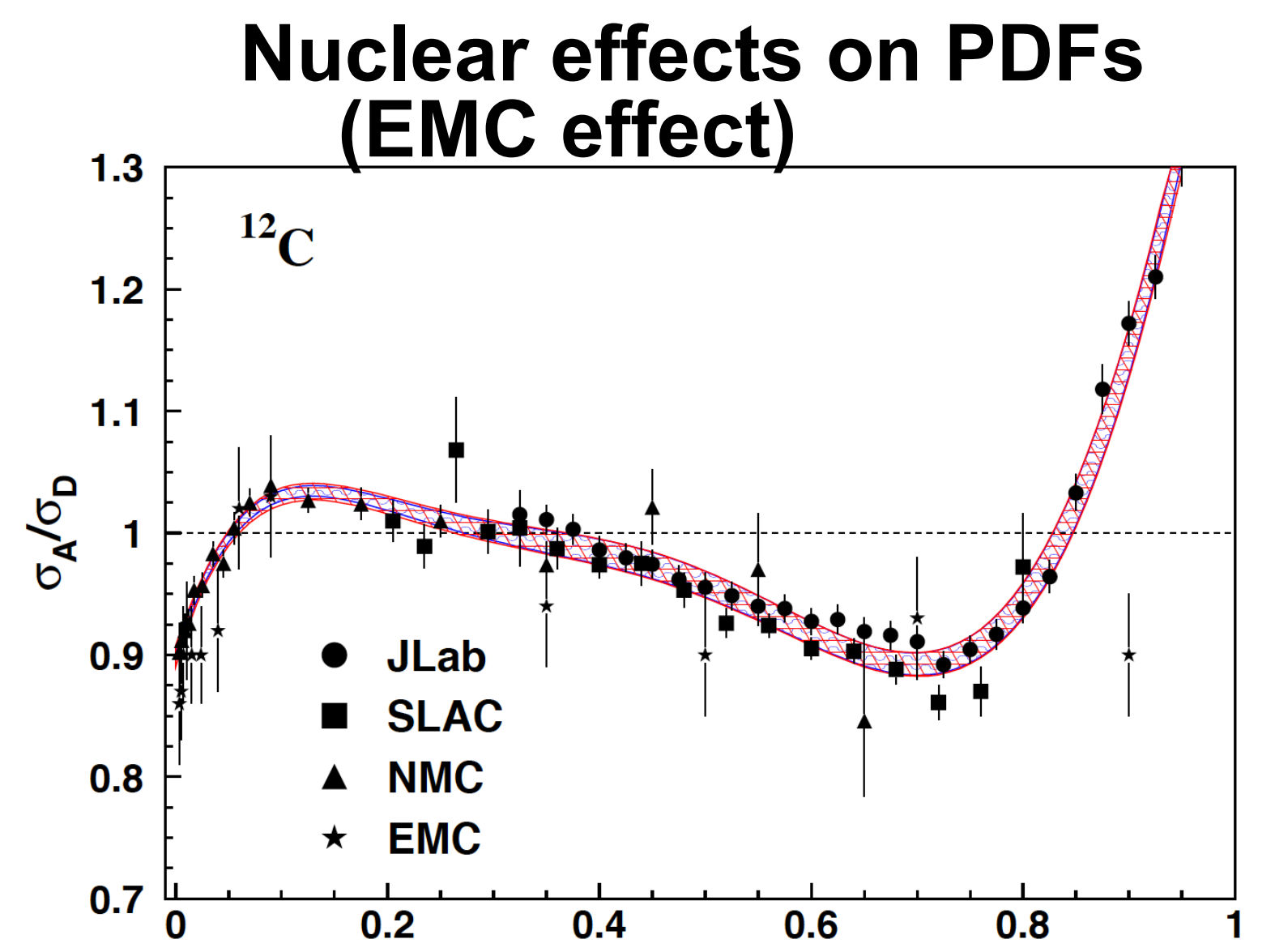

Malace et al, Int. J. Mod. Phys. E 23 (2014) 


\section{QCD constraints on PDFs}

Matrix elements of local operators determine momentum fractions carried by each quark flavor and gluons

$\langle x\rangle_{q}^{(h)} p_{\mu} p_{\nu}=\left\langle h\left|\bar{q} \gamma_{\{\mu} \overleftrightarrow{D}_{\nu\}} q\right| h\right\rangle$
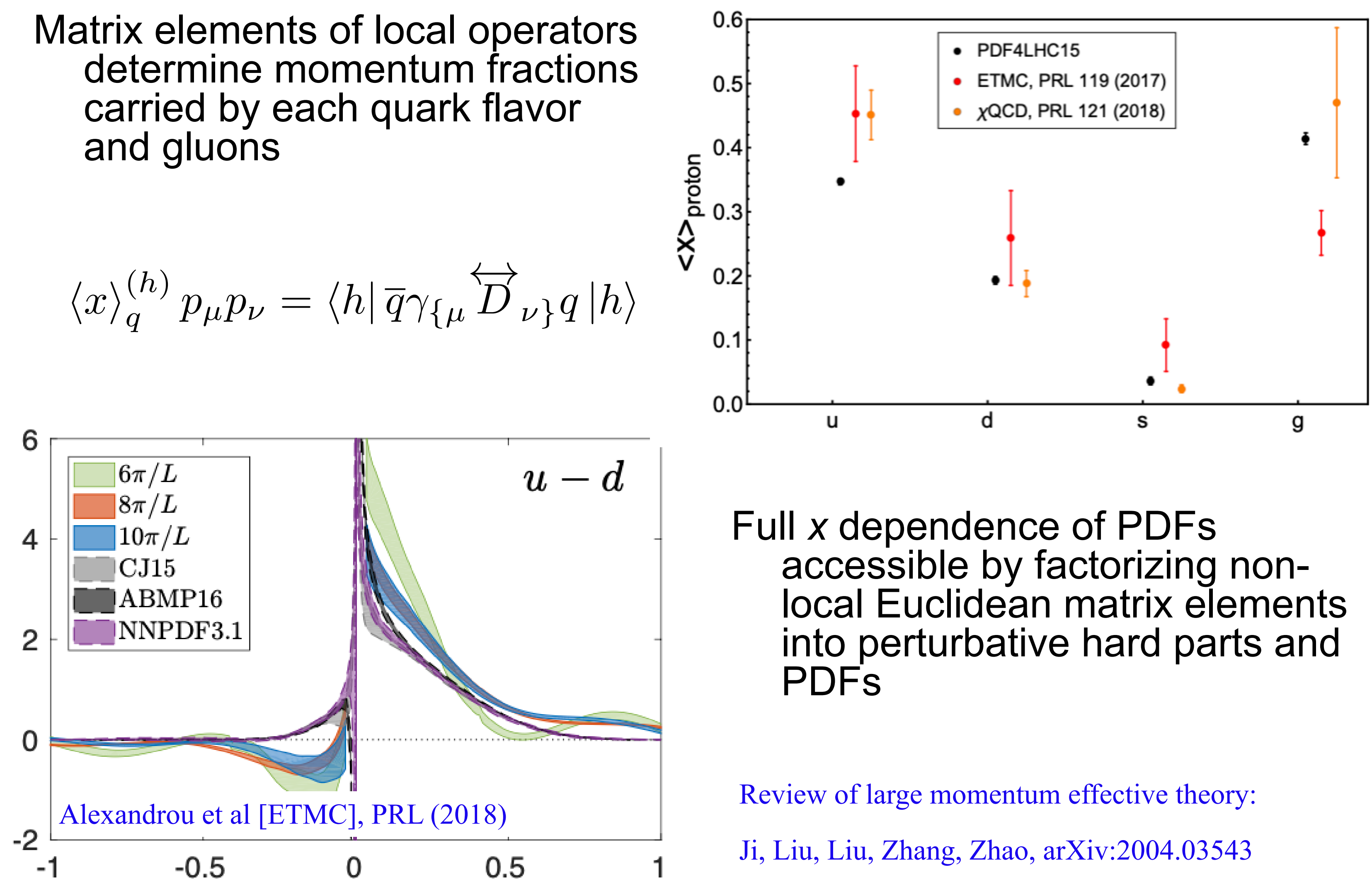

Full $x$ dependence of PDFs accessible by factorizing nonlocal Euclidean matrix elements into perturbative hard parts and PDFs

Review of large momentum effective theory:

Ji, Liu, Liu, Zhang, Zhao, arXiv:2004.03543 


\section{Quarks in nuclei from QCD}

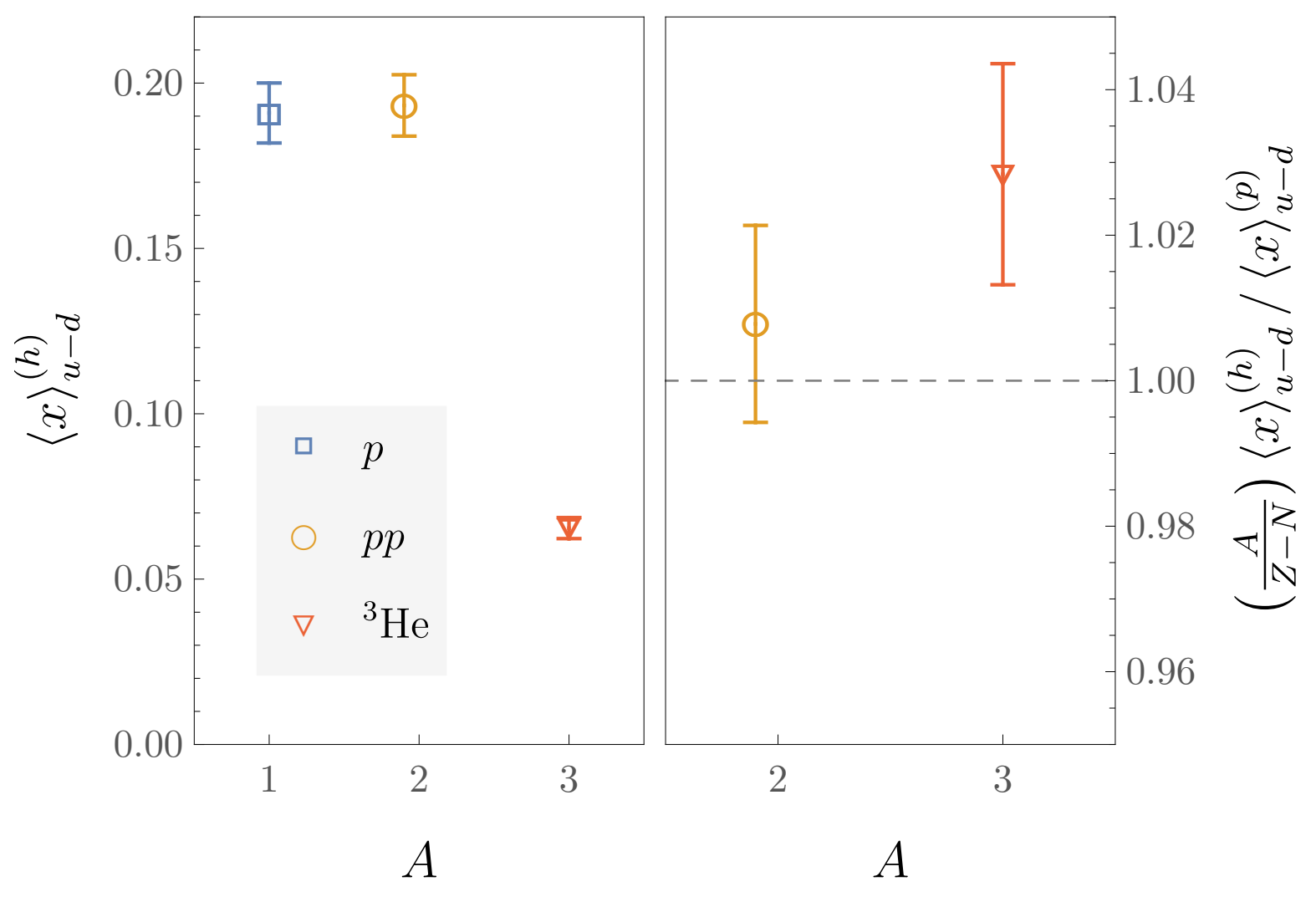

Matrix elements of same operators in nuclear states determine momentum fractions of quarks and gluons in nuclei

Winter et al [NPLQCD], PRD 96 (2017)

Detmold et al [NPLQCD], arXiv:2009.05522

Results used to constrain EFT, extrapolate to physical results

$$
N^{\dagger} \tau^{3} N+\alpha_{3, n} N^{\dagger} \tau^{3} N N^{\dagger} N+\ldots
$$

Flavor dependence of nuclear PDFs is poorly known experimentally, first LQCD + EFT results already provide non-trivial constraints on nuclear PDFs

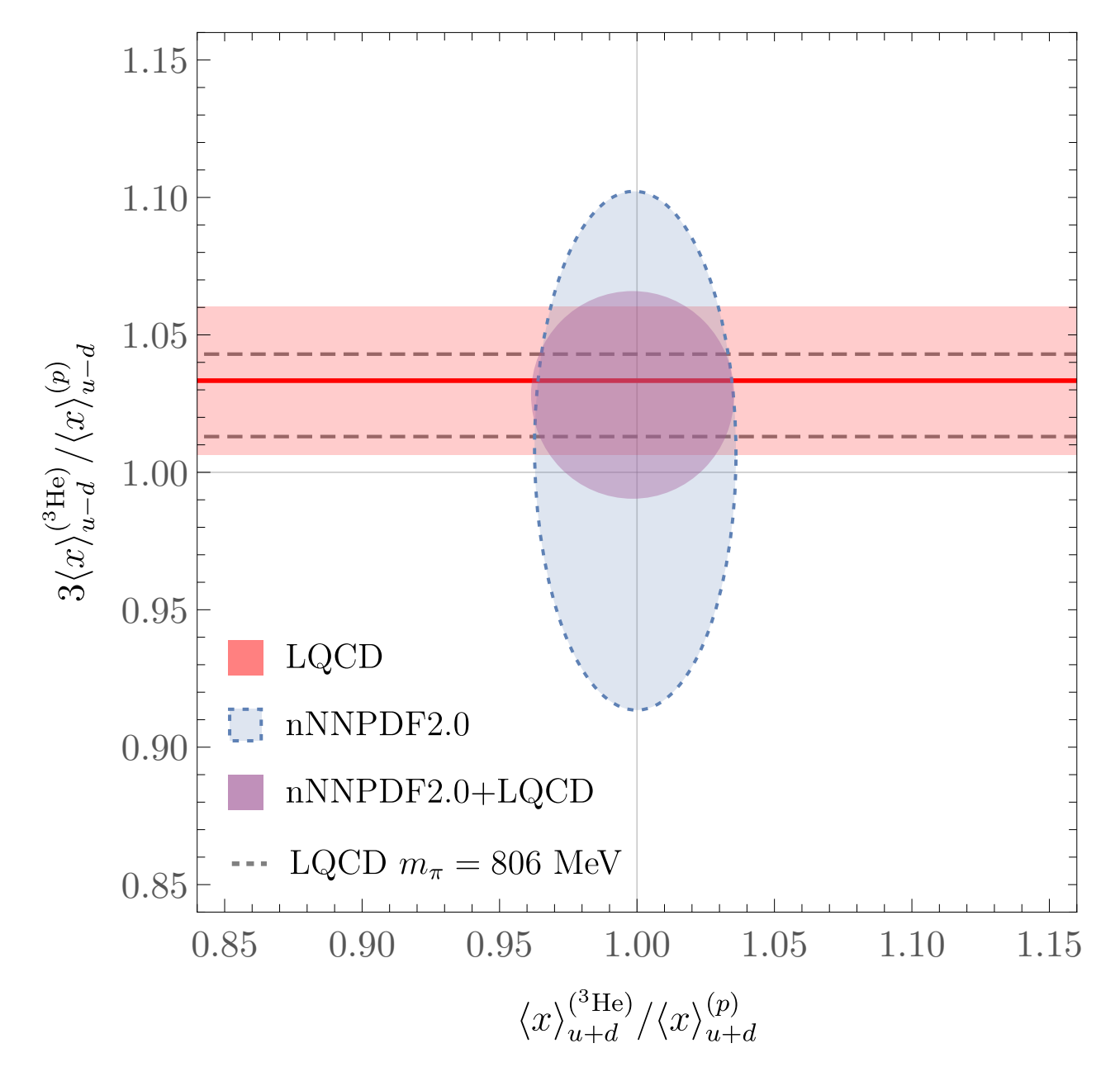




\section{Conclusions}

Describing $\nu A$ scattering from the Standard Model requires control of QCD over a wide range of scales and physics processes

Challenging but possible through lattice QCD + nuclear many body methods

\begin{abstract}
Nucleon form factors
Pion production

Two-body currents

PDF constraints
\end{abstract}
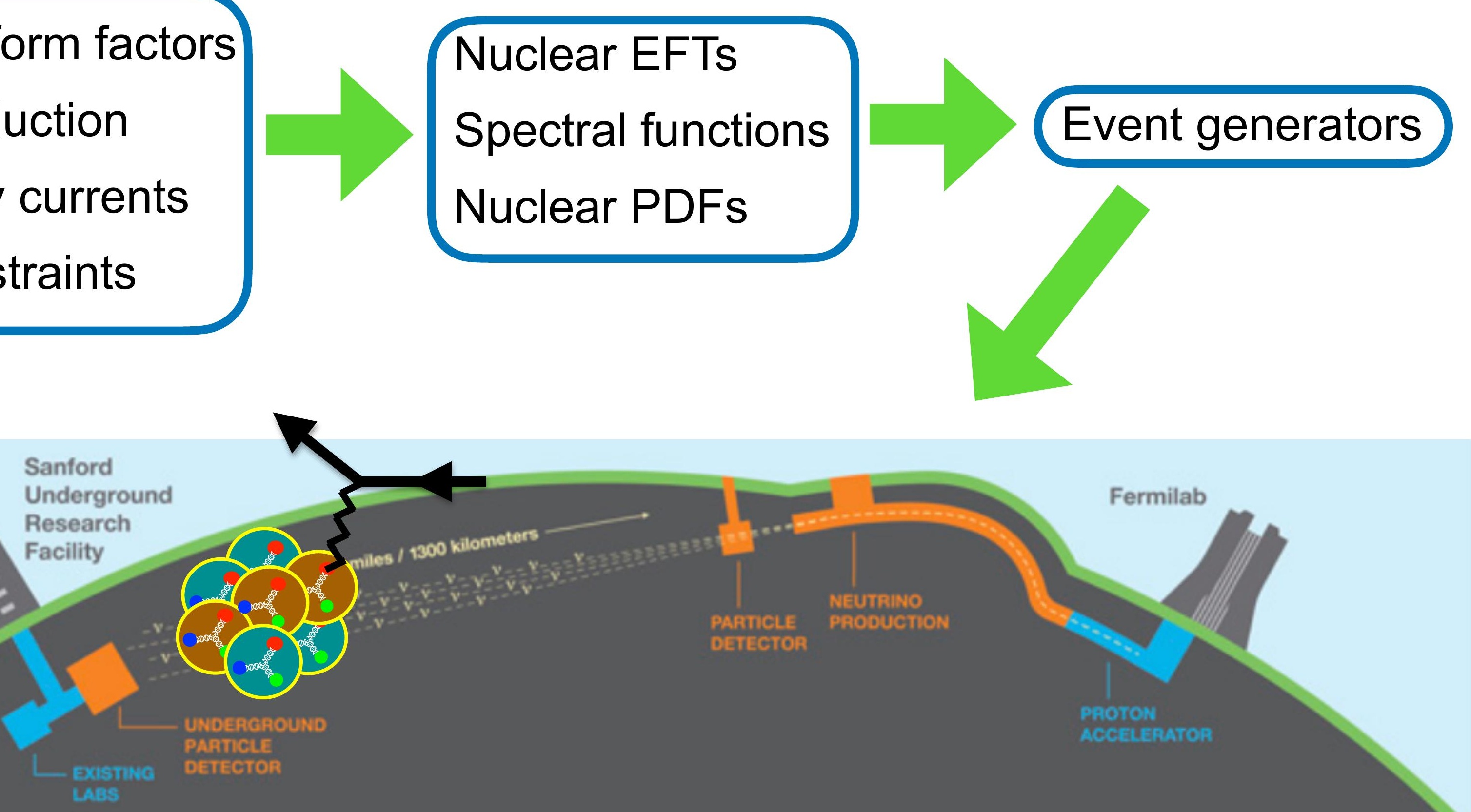